alg-geom/9612012

BONN-TH-96-13

\title{
SCALING BEHAVIOR ON THE SPACE OF CALABI-YAU MANIFOLDS
}

\author{
R.SCHIMMRIGK 田 \\ Physikalisches Institut, Universität Bonn \\ Nussallee 12, 53115 Bonn
}

\begin{abstract}
Recent work is reviewed which suggests that certain universal quantities, defined for all CalabiYau manifolds, exhibit a specific behavior which is not present for general Kähler manifolds. The variables in question, natural from a mathematical perspective, are of physical importance because they determine aspects of the low-energy physics in four dimensions, such as Yukawa couplings and threshold corrections. It is shown that these quantities, evaluated on the complete class of Calabi-Yau hypersurfaces in weighted projective 4-space, exhibit scaling behavior with respect to a new scaling variable.
\end{abstract}

To APpear in Mirror Symmetry II

${ }^{\diamond}$ netah@avzw02.physik.uni-bonn.de 
Introduction. A longstanding question in string theory concerns the structure of the space of groundstates. Much insight has been gained over the past years into some of its salient properties for (2,2)-supersymmetric vacua 1. Most of these results, such as the discovery of mirror symmetry [1] and the fact that different Calabi-Yau manifolds are connected through singular configurations [2], focus on the detailed geometry of the moduli space of individual internal manifolds 9 . Relevant in this context is the generation-antigeneration structure of the heterotic string, encoded in the two nontrivial Hodge numbers $\left(h^{(1,1)}, h^{(2,1)}\right)$ of the Calabi-Yau space. These topological numbers provide a first parametrization of the space of string vacua and mirror symmetry, an operation that exchanges these two numbers, provides a profound technique via which it is possible to extract physical information about individual ground states.

What mirror symmetry has not provided, however, is a framework which leads to insight into the more detailed structure of the space of string vacua and which will enable us, eventually, to address problems like the vacuum degeneracy. In the absence of a physical 'theory of moduli space' what is needed is some concrete information about this space, which we might hope to be encoded in some numerical characteristics, much like data from an experiment. Such numerical information is expected to be useful in providing a guideline as to what exactly it is that a future theory of moduli space has to explain. In ref. [3] it has been shown that such characteristics exist in the form of scaling exponents, thereby taking the first steps toward a quantitative understanding of the moduli space of the supersymmetric closed string. The scaling behavior defined by these exponents is specific to Calabi-Yau manifolds and does not exist for general projective varieties.

From a mathematical perspective the results of [3] form part of a long tradition in algebraic geometry, which runs under the heading 'geography of manifolds' and which reaches back at least as far as the last century. As will become clear in the course of this review the analysis of [3] is reminiscent of Noether's investigation of algebraic surfaces and represents a continuation of the work of Wilson [4] in a slightly different context.

Finally, we will have opportunity to comment on recent results [5] regarding the classification of Calabi-Yau manifolds using the Fujita $\Delta$-index.

The Variables. The quantities which will be focused on in the following are based on the existence of a universal structure which exists on every Calabi-Yau manifold by virtue of the fact

\footnotetext{
${ }^{1}$ In this paper the focus will be on the space of Calabi-Yau manifolds.

${ }^{2}$ Or their field theoretic counterparts in the framework of Landau-Ginzburg theories or conformal field theories.
} 
that they are projective, i.e. they are described by equations in a particular type of compact, complex, Kähler manifold, so-called projective spaces. On such spaces there exists a natural structure, the so-called hyperplane bundle, denoted by $L$, which can be restricted to the CalabiYau manifold embedded in the ambient projective space. This bundle is of interest for physics because its first Chern class $c_{1}(L)$ leads to a universal massless mode, present in every Calabi-Yau manifold, which parametrizes one of the antigenerations observed in four dimensions. Moreover, on a three-complex dimensional Calabi-Yau manifold, the degree of this line bundle, defined as

$$
L^{3} \equiv \int_{M} c_{1}^{3}(L)
$$

affords an interpretation as a Yukawa coupling $\kappa_{L} \equiv L^{3}$ of the antigeneration associated to $L$. Thus $L^{3}$ determines the strength of the Yukawa coupling of the corresponding four-dimensional mode. A second characteristic of any line bundle is the number of its global functions, denoted by $h_{L}$. For every Calabi-Yau manifold the line bundle $L$ thus leads to a pair of quantities $\left(L^{3}, h_{L}\right)$ which can be viewed as coordinates on the space of Calabi-Yau manifolds. The question arises whether these new coordinates allow us to draw some type of 'phase diagram' for the spaces of interest, i.e. whether they provide a new sort of structure theorem for string vacua, furnishing a new 'geographical map'.

A final quantity of interest which is induced by the line bundle $L$ is

$$
L \cdot c_{2} \equiv \int_{M} c_{1}(L) \wedge c_{2}(M)
$$

where $c_{2}(M)$ is the second Chern class of the Calabi-Yau 3-fold. The physical interpretation of this quantity has been uncovered in ref. [6], where it was shown that the generalized index

$$
\mathcal{F}=\frac{1}{2} \int \frac{d^{2} \tau}{\operatorname{Im} \tau} \operatorname{Tr}\left[(-1)^{F} F_{L} F_{R} q^{H_{L}} \bar{q}^{H_{R}}\right]
$$

introduced in [7], contains information about the threshold corrections of the gauge couplings in string theory (see also [8]) and that it is the key for the understanding of quantum mirror symmetry at one loop. Here the integral is over the fundamental domain of the moduli space of the torus, $F_{L, R}$ denote the left and right fermion numbers and the trace is over the Ramond sector for both the left- and right-movers [5. In lowest order this index essentially reduces to $\frac{1}{24} \int_{M} K \wedge c_{2}(M)$, where $K$ is the Kähler form of the manifold. Hence the second Chern class evaluated on $L$, (2) , defines the universal contribution $\mathcal{F}_{L}^{\uparrow} \equiv L \cdot c_{2} / 24$ to the large volume limit of this partition function.

\footnotetext{
${ }^{3}$ The contribution of the ground states of the supersymmetric Ramond sector to $\mathcal{F}$ has to be deleted in order for the integral to converge.
} 
A useful fact is that the three variables $L^{3}, h_{L}, L \cdot c_{2}$ are not all independent because of the theorem of Hirzebruch-Riemann-Roch, which relates the Euler number defined by the counting problem

$$
\chi(M, L)=\sum_{p=0}^{n}(-1)^{p} \operatorname{dim} \mathrm{H}^{p}(M, L)
$$

to an integral over the manifold

$$
\chi(M, L)=\int_{M} e^{c_{1}(L)} \wedge \operatorname{Td}(M),
$$

where $\operatorname{Td}(M)$ is the Todd class of the manifold. For Calabi-Yau 3-folds this leads to $\chi(M, L)=$ $\frac{1}{6} L^{3}+\frac{1}{12} L \cdot c_{2}$ and hence

$$
L^{3}=6 h_{L}-\frac{1}{2} L \cdot c_{2}
$$

Finally, we quote, for future use, the definition of the Fujita index 99 which can be defined for a variety of arbitrary dimension $n$ as

$$
\Delta=n+L^{n}-h_{L}
$$

This index has proven useful in the classification of polarized varieties in particular when it assumes small values.

The Class. A class of Calabi-Yau spaces that is particularly amenable to an analysis in terms of the variables $L^{3}, L \cdot c_{2}$, and $h_{L}$ is furnished by hypersurfaces embedded in weighted projective space $\mathbb{P}_{\left(k_{1}, \ldots, k_{5}\right)}$. The complete class of such manifolds, consisting of 7,555 configurations, has been constructed in [10, 11]. The natural candidate for a line bundle on such spaces is the pullback of the weighted form of the hyperplane bundle $L \equiv \mathcal{O}_{\mathbb{P}_{\left(k_{1}, \ldots, k_{5}\right)}^{(k)}}^{(k)}$ defined on the weighted ambient space, where 12, 13.

$$
k=l c m\left\{\left\{\operatorname{gcd}\left(k_{i}, k_{j}\right) \mid i, j=1, . ., 5 ; i \neq j\right\} \cup\left\{k_{i} \mid k_{i} \text { does not divide } \sum_{i=1}^{5} k_{i}\right\}\right\} .
$$

The restriction of $L$ from the weighted ambient space to the embedded Calabi-Yau manifold $M$ induces an antigeneration which will also be denoted by $L$. The Yukawa coupling $\kappa_{L}$ of this antigeneration leads, in the large volume limit, to the expression of the degree of $L$

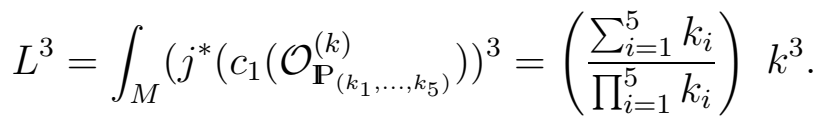

The number of global functions of the bundles $L=\mathcal{O}_{\mathbf{P}_{\left(k_{1}, \ldots, k_{5}\right)}}^{(k)}$ can be obtained as

$$
h_{L}=\left.\frac{1}{k !} \frac{\partial^{k}}{\partial t^{k}}\left(\frac{\left(1-t^{\sum_{i=1}^{5} k_{i}}\right)}{\prod_{i=1}^{5}\left(1-t^{k_{i}}\right)}\right)\right|_{t=0}
$$


and therefore we can determine the Fujita index and also, via the Hirzebruch-Riemann-Rochrelation the second Chern class evaluated on the hyperplane bundle. In the following these variables will be used to explore the structure of the moduli space of Calabi-Yau manifolds.

\section{The Results.}

The Yukawa coupling: For arbitrary projective manifolds the two numbers $L^{3}$ and $h_{L}$ are independent, completely uncorrelated, quantities. Hence in a diagram of these numbers we might expect a random distribution of data points, with no obvious pattern. The actual computation for Calabi-Yau manifolds, the results of which are shown in Figure 1, however uncovers an unexpected simplicity.

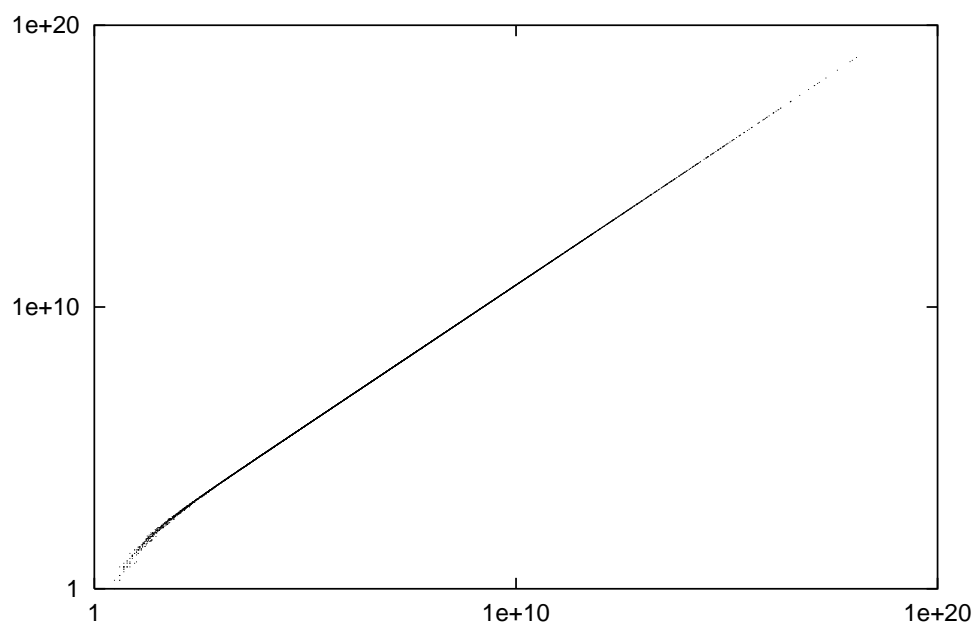

Figure 1: The degree $L^{3}$ versus $h_{L}$.

The first thing to notice is the emergence of a well defined boundary which is described by a simple relation: all varieties in the class of Calabi-Yau 3-folds embedded in weighted $\mathbb{P}_{\left(k_{1}, \ldots, k_{5}\right)}$ satisfy the inequality

$$
L^{3} \leq 2\left(3 h_{L}-8\right)
$$

This is in contrast to mirror symmetry where the boundary of the mirror diagram (see the first reference in [1] ) does not yield to such an easy description, even though it is also well defined.

What is rather unexpected is that as the dimensions $h_{L}$ increase for the individual CalabiYau hypersurfaces the Yukawa couplings approach the upper limit $L_{a s}^{3} \equiv 2\left(3 h_{L}-8\right)$ very quickly,

\footnotetext{
${ }^{4}$ The weaker limit $\kappa_{L}<6 h_{L}$ follows from the relation of Hirzebruch-Riemann-Roch and the positivity of the second Chern class.
} 
with very little scattering. Furthermore they do so according to a power law. This can be seen most clearly from Figure 2, which contains the distances $\mathcal{D} \equiv\left(L_{a s}^{3} / L^{3}-1\right)$ of the degree of $L$ from $L_{a s}^{3}$ as a function of $h_{L}$.

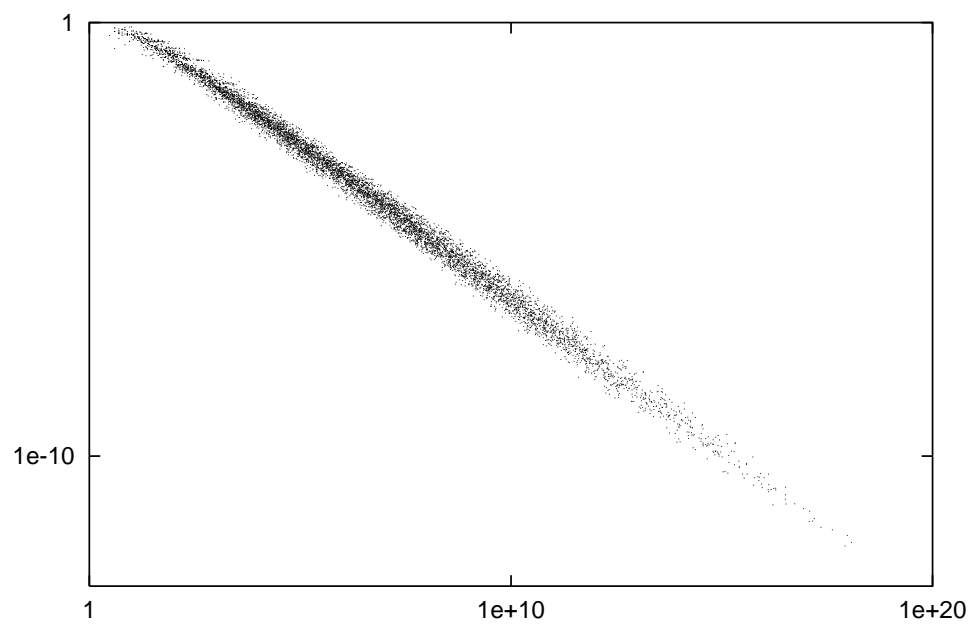

Figure 2: $\mathcal{D}$ versus $h_{L}$.

The result shows clearly that the distance variable $\mathcal{D}$ exhibits a scaling behavior with approximate exponents $A=5$ and $\alpha=0.7$, determined by a least square fit. Solving this scaling relation leads to the functional dependence of the degree of the hyperplane bundle on $h_{L}$. These results can be summarized more precisely in the following

Theorem 1. All varieties in the class of CY 3-folds embedded in weighted projective 4-space $\mathbb{P}_{\left(k_{1}, \ldots, k_{5}\right)}$ satisfy the inequality

$$
L^{3} \leq 2\left(3 h_{L}-8\right)
$$

The distance $\mathcal{D} \equiv \frac{2\left(3 h_{L}-8\right)}{L^{3}}-1$ from the asymptotic line $L_{\text {as }}^{3} \equiv 2\left(3 h_{L}-8\right)$ scales like

$$
D \sim A\left(h_{L}\right)^{-\alpha}
$$

with $A \sim 5$ and $\alpha \sim 0.7$. Thus the functional dependence of the degree on $h_{L}$ is given by

$$
L^{3} \sim \frac{L_{a s}^{3}}{1+A\left(h_{L}\right)^{-\alpha}}
$$

\section{Remarks.}

- Physically therefore the Yukawa coupling $\kappa_{L} \equiv L^{3}$ behaves like an order parameter with the dimension $h_{L}$ assuming the rôle of the scaling variable. 
- The degree $L^{3}$ and the dimension $h_{L}$ take values over an enormously wide range of values, unprecedented in Calabi-Yau physics, spanning 18 orders of magnitude. This will propagate via the Hirzebruch-Riemann-Roch relation to $L \cdot c_{2}$ and lead to an enormous variation for the threshold corrections of these string vacua (see remark below).

- The power law behavior (14) for the coupling provides some measure of simplicity of CalabiYau manifolds: the larger the value of the scaling variable $h_{L}$, the more accurate the prediction of the Yukawa coupling via (14). Since, very roughly, the magnitude of the scaling variable is determined by the degree of complexity of the singular sets of the varieties that need to be resolved, spaces with more complicated resolution geometry are in fact simpler when it comes to scaling. This phenomenon is rather different from the conventional point of view which considers smooth projective hypersurfaces as the simplest type of manifolds.

- Finally, it is easy to see that the scaling behavior (13), (14) is a characteristic of Calabi-Yau manifolds, not of general projective 3-folds, not to mention general 3-folds: consider the hypersurfaces $\mathbb{P}_{4}[d]$ of degree $d$ embedded in ordinary projective 4 -space. For this (infinite) class of manifolds one finds $L^{3}=d, h_{L}=5, L \cdot c_{2}=d\left(d^{2}-5 d+10\right)$.

The second Chern class. Over the last years a confluence of ideas has occured in physics and mathematics toward a further physically important quantity. Wilson [4], on the one hand, has reemphasized the importance of the linear form on the Picard group, defined by the second Chern class $c_{2}$, for purposes of classification, a fact first recognized by Wall. Bershadsky, Cecotti, Ooguri, and Vafa, on the other, have found that the generalized index of [7] reduces in the large volume limit essentially to $\mathcal{F}_{L}^{\uparrow}=L \cdot c_{2} / 24$, and have shown that this quantity arises in the 1 -loop quantum mirror expansion of the Ray-Singer torsion, as well as in the threshold corrections of the gauge couplings (see also [B]). Thus the pair $\left(L^{3}, L \cdot c_{2}\right)$ provides two coordinates on the moduli space of Calabi-Yau manifolds with immediate physical meaning. To investigate a possible relation between these quantities is of particular interest since they arise in two different perturbation expansions of the string - the $\sigma$-model expansion at string tree level and the string loop expansion.

Wilson [4] has observed that for the degree $L^{3}$ and $L \cdot c_{2}$ an inequality $L \cdot c_{2} \leq 10 L^{3}$ is obtained for models whose Fujita index $\Delta$ is greater than two. The analysis of the present class of weighted Calabi-Yau hypersurfaces shows that for all but 11 spaces the Fujita index is always larger than two. Hence Wilson's observation shows that except for those sixteen spaces the inequality above 
does hold in this class, i.e. in physics notation

$$
\frac{\mathcal{F}_{L}^{\uparrow}}{\kappa_{L}} \leq \frac{5}{12} .
$$

No further information about the population below this upper limit is known.

The unexpected consequence of the analysis above of all Calabi-Yau hypersurfaces in weighted projective 4-space is that the vast majority of these vacua does not come even close to the upper limit (15) but instead takes values in a narrow region far below that line. For large values of $L$. the Yukawa couplings lie more than ten orders of magnitude below the upper limit provided by Wilson's observation. Furthermore the results, as shown in Figure 3, uncover a definite correlation between the degree of $L$ and $L \cdot c_{2}$.

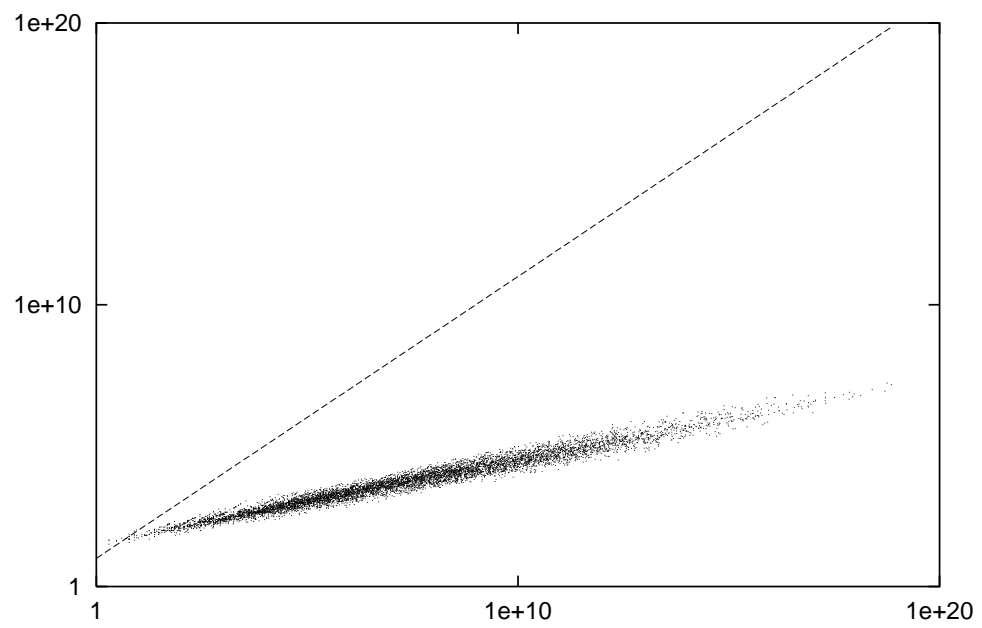

Figure 3: $L \cdot c_{2}$ versus $L^{3}$. The dotted line represents Wilson's upper limit.

An analysis similar to the one discussed previously leads to the following

Theorem 2. In the class of Calabi-Yau hypersurfaces in weighted projective space $\mathbb{P}_{\left(k_{1}, \ldots, k_{5}\right)}$ the product $L \cdot c_{2}$ scales like

$$
L \cdot c_{2} \sim B \kappa_{L}^{\beta}
$$

with approximate values

$$
B=36, \quad \beta=0.29 \text {. }
$$

Remark. As mentioned above, the range of $L \cdot c_{2}$ is huge, resulting in vast range of low-energy 
effective GUT mass scales [6]

$$
M_{\mathrm{GUT}}^{\mathrm{eff}}=M_{\mathrm{GUT}} \exp \left(\frac{1}{b} \int_{M} K \wedge c_{2}(M)\right),
$$

where $b=54+3\left(h^{(1,1)}+h^{(2,1)}\right.$.

The Fujita index: From the definition of this index it is clear that it will behave precisely like the degree of the hyperplane bundle when viewed as a function of $h_{L}$.

Of particular interest to mathematicians have been manifolds with low values of the $\Delta$-genus. In [5] all smooth Calabi-Yau manifolds with $\Delta \leq 2$ have been classified. In the set of all CalabiYau hypersurfaces in weighted projective space there are only 4 smooth members. The results of [3] lead to an immediate generalization of Oguiso's analysis and show that the class of manifolds with low Fujita index broadens considerably when resolutions are admitted. Table 1 contains the weighted configurations with $\Delta \leq 2$ together with some of their pertinent properties.

\begin{tabular}{||lccccccc||}
\hline \hline Manifold & $\Delta$ & $L^{3}$ & $h_{L}$ & $L \cdot c_{2}$ & Euler number & $h^{(1,1)}$ & $h^{(2,1)}$ \\
\hline \hline $\mathbb{P}_{(1,1,1,2,5)}[10]$ & 1 & 1 & 3 & 34 & -288 & 1 & 145 \\
$\mathbb{P}_{(1,2,2,2,7)}[14]$ & 1 & 2 & 4 & 44 & -240 & 2 & 122 \\
$\mathbb{P}_{(1,1,1,1,4)}[8]$ & 1 & 2 & 4 & 44 & -296 & 1 & 149 \\
$\mathbb{P}_{(1,2,2,3,4)}[12]$ & 2 & 2 & 3 & 32 & -144 & 2 & 74 \\
$\mathbb{P}_{(1,3,3,3,5)}[15]$ & 2 & 3 & 4 & 42 & -144 & 3 & 75 \\
$\mathbb{P}_{(1,2,3,3,9)}[18]$ & 2 & 3 & 4 & 42 & -192 & 3 & 99 \\
$\mathbb{P}_{(1,1,1,1,2)}[6]$ & 2 & 3 & 4 & 42 & -204 & 1 & 103 \\
$\mathbb{P}_{(1,1,2,2,6)}[12]$ & 2 & 4 & 5 & 52 & -252 & 2 & 128 \\
$\mathbb{P}_{(1,2,3,12,18)}[36]$ & 2 & 6 & 7 & 72 & -360 & 5 & 185 \\
$\mathbb{P}_{(1,1,2,8,12)}[24]$ & 2 & 8 & 9 & 92 & -480 & 3 & 243 \\
$\mathbb{P}_{(1,1,1,6,9)}[18]$ & 2 & 9 & 10 & 102 & -540 & 2 & 272 \\
\hline \hline
\end{tabular}

Table 1: All Calabi-Yau manifolds embedded in weighted $\mathbb{P}_{4}$ with $\Delta \leq 2$.

This indicates that there should be still more to explore once one is able to probe more general weighted complete intersections of hypersurfaces in toric varieties and their generalizations.

In summary, the results presented above suggest that the degree $L^{3}$ and the universal lowest order part $L \cdot c_{2}$ of the free energy should be interpreted as order parameters for which the number 
$h_{L}$ of global functions behaves like a scaling variable. The scaling phenomena described above provide specific power laws with a particular set of exponents that any future physical theory of moduli space of the heterotic string will have to explain. Thus our scaling laws furnish the first quantitative insight into the structure of the string configuration space.

The present situation is not without historical precedent in physics: it is reminiscent of Bjorken's results many years ago when he argued that a certain scaling behavior should manifest itself in deep inelastic electron-nucleon scattering. Our scaling can in fact be presented in a form which is very similar to the original data of these historical results. Define a function $\left(h_{L}\right)^{-1} \kappa_{L}$ and consider its dependence on the scaling variable $h_{L}$. The result, which is displayed in Figure 4 , looks very much like the experimental results of the SLAC group for $\nu W_{2}\left(\nu, q^{2}\right)$.

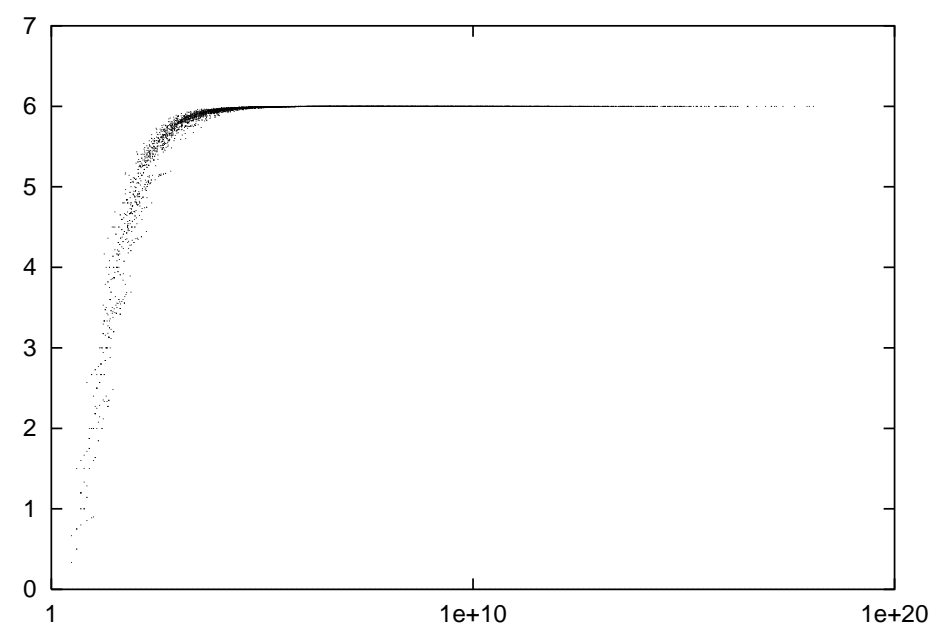

Figure 4: $\left(h_{L}\right)^{-1} \kappa_{L}$ versus $h_{L}$.

What is needed, then, is the analog of Feynman's quark-parton picture, or perhaps rather the analog of the QCD explanation of, say, the momentum fraction of the proton carried by the quarks.

Tantalizing questions remain: An immediate one is whether the exponents $\alpha, \beta$ are universal or specific to our class of Calabi-Yau hypersurfaces in weighted projective 4 -space. There are two natural avenues for further exploration. The simpler of the two is provided by the toric framework of ref. [14] which has been shown [15] to include all Calabi-Yau hypersurfaces in weighted projective 4-space. The second direction involves a natural generalization of the framework of Calabi-Yau compactification, motivated by mirror symmetry. In [16] a construction has been introduced which provides an embedding of Calabi-Yau spaces into a larger class of a special type 
of Fano manifolds, varieties whose positive first Chern class satisfies a particular quantization condition. It was shown in [16] that even though these special Fano manifolds are not consistent ground states themselves they do contain information about $(2,2)$ supersymmetric string vacua. It was furthermore demonstrated in ref. [17 that such manifolds also lead to the correct behavior of the Yukawa couplings and in ref. [18] that the framework of [16] lends itself to a toric analysis, generalizing the toric mirror construction of [14]. A natural question is whether our scaling behavior persists in these more general settings as well. Finally, the computations presented here are of lowest order in perturbation theory and an interesting problem is to explore the consequences of including higher order corrections.

\section{Acknowledgment}

It is a pleasure to thank Philip Candelas, Dimitrios Dais, Xenia de la Ossa, Ed Derrick, Michael Flohr, Ariane Frey, Jerry Hinnefeld, Vadim Kaplunovsky, Jack Morse, Werner Nahm, Steve Shore, and especially Andreas Honecker, Monika Lynker and Katrin Wendland for discussions. I'm grateful to the Theory Group at the University of Texas at Austin, the Department of Physics at Indiana University at South Bend, and Simulated Realities Inc., Austin, TX for hospitality.

\section{References}

[1] P.Candelas, M.Lynker and R.Schimmrigk, Nucl.Phys. B341(1990)383

M.Lynker and R.Schimmrigk, Phys.Lett. B249(1990)237

B.R.Greene and R.Plesser, Nucl.Phys. B338(1990)15

[2] P.Candelas, A.Dale, C.A.Lütken and R.Schimmrigk, Nucl.Phys. B298(1988)493

[3] R.Schimmrigk, Phys.Lett. B388(1996)60

[4] P.M.H.Wilson, Contemporary Mathematics 162(1994)403

[5] K.Oguiso, J.Fac.Sci.Univ.Tokyo 38(1991)395

[6] M.Bershadsky, S.Cecotti, H.Ooguri and C.Vafa Nucl.Phys. B405(1993)279; Commun.Math.Phys. 165(1994)311

[7] S.Cecotti, P.Fendley, K.Intriligator and C.Vafa, Nucl.Phys. B386(1992)405 
[8] I.Antoniadis, E.Gava and K.S.Narain, Nucl.Phys. B383(1992)93

[9] T.Fujita, Classification Theories of Polarized Varieties, Cambridge University Press 1990

[10] A.Klemm and R.Schimmrigk, Nucl.Phys. B411(1994)559

[11] M.Kreuzer and H.Skarke, Nucl.Phys. B388(1992)113

[12] D.Dais, to appear in Bonner Mathematische Schriften

[13] P.Berglund and S.Katz, Nucl.Phys. B420(1994)289

[14] V.V.Batyrev, J.Alg.Geom. 3(1994)493

[15] P.Candelas, X.de la Ossa and S.Katz, Nucl.Phys. B450(1995)267

[16] R.Schimmrigk, Phys.Rev.Lett. 70(1993)3688; Int.J.Mod.Phys. A11(1996)3049

[17] P.Candelas, E.Derrick and L.Parkes, Nucl.Phys. B407(1993)115

[18] V.V.Batyrev and L.A.Borisov, University of Essen and University of Michigan preprints, 1994 